\title{
Case of sphincter reconstruction and stoma in situ for abdominoperineal resection with rectal cancer: A 34-year experience
}

\author{
jinchun cong ${ }^{1}$ \\ ${ }^{1}$ Shengjing Hospital of China Medical University
}

July 30,2020

\begin{abstract}
Sphincter reconstruction and stoma in situ is an exploratory surgery for abdominoperineal resection of rectal cancer; its functional outcome is a major concern. This is a rare case of long-term survival with unilateral gluteus maximus fasciculus reconstruction, in a patient with the ability to control defecation and distinguish gas.
\end{abstract}

\section{Keywords}

Rectal cancer, abdominoperineal resection, sphincter reconstruction, stoma in situ

\section{Key Clinical Message}

Sphincter reconstruction and stoma in situ is an exploratory surgery for abdominoperineal resection of rectal cancer; its functional outcome is a major concern. This is a rare case of long-term survival with unilateral gluteus maximus fasciculus reconstruction, in a patient with the ability to control defecation and distinguish gas.

\section{Introduction}

In 1908, Miles described abdominoperineal resection (APR) for carcinoma of the lower third of the rectum and diversion by permanent abdominal colostomy. In recent decades, the frequency of APR use has reduced due to advances in neoadjuvant radiochemotherapy and surgical techniques; nevertheless, a significant number of patients with very low rectal cancer still undergo APR for curative intended surgery. ${ }^{1}$ Several methods of sphincter reconstruction and stoma in situ, following conventional APR, have been described to avoid permanent abdominal colostomy and improve quality of life. ${ }^{2-5}$ In the $1980 \mathrm{~s}$, similar surgeries were performed over a 10-year period in China, but no long-term results, particularly functional outcomes, were reported.

\section{Case History/Examination}

We present the case of a 66-year-old man who was diagnosed with rectal cancer in 1986 and underwent APR, sphincter reconstruction, and stoma in situ. The patient recalled that antidiarrhea drugs were needed to control defecation for nearly one year after the surgery. He gradually overcame his dependence on these drugs and developed autonomous control of defecation. For more than 30 years, he maintained a strict diet. The feces in most cases were separate hard lumps, and he had little or no diarrhea. The patient's bowel movement has been regular for the last 30 years, almost fixed at 6 a.m., 5 p.m., and 10 p.m. every day, for approximately $10 \mathrm{~min}$ each time, without continued sensation. The patient was able to control defecation within 10 minutes of noticing the need to defecate and any time frame beyond period is not known as he always had access to a toilet. The patient can recognize gas; although usually, he has no control over the passage of gas. The patient has been able to distinguish between gas and defecation for the last 30 years. Urination has been normal after the surgery; regrettably, the patient refused to answer any questions 
regarding sexual function. In the last 5 years, the stomal colon mucosa gradually prolapsed, which resulted in the sensation of bulging and presence of a foreign body; thus, the patient became increasingly intolerant of the symptoms and chose to undergo removal of the prolapsed mucosa.

The body mass index of the patient was $24.5 \mathrm{~kg} / \mathrm{m}^{2}$. He had no risk of malnutrition according to subjective global assessment. He had no history of drug use, except irregular use of hypotensive drugs for familial hypertension. Routine hematological and biochemical investigations showed no remarkable findings. The appearance of the neo-anus (see Figure 1 and Figure 2) shows no change in the perineal hernia or peristomatitis. Skin pigmentation appeared within $2 \mathrm{~cm}$ around the neo-anus and the patient could not remember when the change began; the mucosal prolapse was approximately $3 \mathrm{~cm}$ and appeared thickened and rough. The neo-anorectum was relaxed on digital examination; muscle contraction could be obviously felt at a distance of 2 to $3 \mathrm{~cm}$ from the anal skin when the patient was told to clamp the neo-anus, and the contraction force caused a feeling of blockage when pulling out the finger, lasting for approximately $30 \mathrm{~s}$. The rectum was obviously biased to the right side above this muscle bundle. The patient underwent a computed tomography (CT) examination; unfortunately, he refused to undergo further rectal manometry and magnetic resonance imaging. CT revealed that the rectum was biased to the right side in the pelvis. A bunch of muscles was visible from the right gluteus maximus and was unbroken surround the rectum; the gluteus maximus fasciculus was most apparent in front of the rectum (Figure 3) and was slightly slender at the back (Figure 4), with the inner part close to the coccyx. Referencing previous surgical records, an approximate $3 \mathrm{~cm}$ wide gluteus maximus fasciculus was repositioned from the free end on the outside layer separate from the right gluteus maximus; therefore, the fasciculus passed from front to behind around the rectum, and was attached with an anococcygeal ligament inside and back to the right gluteus maximus on the outside. The thickened mucous membrane in the neo-anus was easily observed on CT (Figure 5), which we suspected was involved in the control of defecation. The patient confirmed that the progressive prolapsed mucosa developed only in the last 5 years and did not affect the control of defecation or gas.

To avoid changes in the bowel movement of the patient, no intestinal preoperative preparation was performed, and the surgery was performed under sacral anesthesia. To avoid damaging the reconstructed sphincter, the separation was only performed up to the level of subcutaneous layer. After resecting the prolapsed mucosa, the colic full-thickness wall was fixed to skin by an interrupted suture (Figure 6). The patient was discharged 2 days after the operation without any complications or changes in bowel movement. The patient was followed up by telephone one month later. The sensation of bulging and presence of a foreign body had disappeared, and the ability to control the passage of gas and defecation was not affected.

\section{Discussion}

In this case report, we describe the case of a man who underwent APR with sphincter reconstruction and stoma in situ. Thirty-four years after the surgery, the patient had regular bowel habits, defecation could be controlled within a time limit, and he could distinguish between gas and feces.

Stoma in situ, also known as perineal colostomy. The original intention to perform this procedure was to suit the patient's psychological state; the second was to construct a neo-anus with the control function. Therefore, a series of sphincter reconstructions is usually performed simultaneously, mainly using skeletal muscle, colonic smooth grafts, and artificial sphincter implants. ${ }^{2-5}$ As a skeletal muscle, the gluteus maximus fasciculus has obvious advantages of convenience and abundant tissue. ${ }^{3}$ Although there is no clear evidence, this approach does not seem to be widely accepted because of scarce literature on this procedure after the 1990s. The biggest question with respect to this sphincter reconstruction surgery is the function, mainly whether the skeletal muscle can maintain a continuous contraction tonus, and second, whether this muscle bundle will slowly become atrophic into a non-functional fibrous structure. This case may be an inspiration to the initial proponents of this surgery because the patient can control defecation and also distinguish between feces and gas in the long term. In our case, defection control also seemed to require good bowel habits, but it is not clear whether the bowel is biased to one side in the pelvis in favor of fecal storage. With respect to distinguishing between gas and feces, it is also unclear how he has compensated since the anus nerve receptor at the end of the terminal was removed, normally patients who have had APR do not have 
this ability..

\section{Conclusion}

In conclusion, reconstruction of the anal sphincter and stoma in situ using unilateral gluteus maximus fasciculus after APR can achieve the desired outcome. The reconstructed sphincter can perform the function of controlling defecation, and the patient can distinguish between gas and feces.

\section{Acknowledgments}

Funding: This work was supported by Natural Science Foundation of Liaoning Province of China (Grant No. 2019-ZD-0747)

\section{Conflict of Interest}

The authors declare that they have no competing interests.

ORCID iD

jinchun cong https://orcid.org/0000-0002-2575-4808

\section{Consent}

Written informed consent was obtained from the patient for publication of this case report and any accompanying images.

\section{Authorship}

Cong $\mathrm{J}$ and Chen $\mathrm{C}$ : were actively involved in the clinical care of the patient.

Cong J: wrote the manuscript.

\section{References}

1.Henry S Tilney, Alexander G Heriot, Sanjay Purkayastha, Anthony Antoniou, Paul Aylin, Ara W Darzi, Paris P Tekkis. 2008.A National Perspective on the Decline of Abdominoperineal Resection for Rectal Cancer. Ann Surg.247:77-84.

2. E Cavina, M Seccia, G Evangelista, M Chiarugi, P Buccianti, A Chirico, M Lenzi, P Bortolotti, G Bellomini, M Arganini, et al. 1987. Construction of a Continent Perineal Colostomy by Using Electrostimulated Gracilis Muscles After Abdominoperineal Resection: Personal Technique and Experience With 32 Cases. Ital J Surg Sci.17:305-14.

3. N Iwai, H Kaneda, T Tsuto, J Yanagihara, T Takahashi.Objective Assessment of Anorectal Function After Sphincter Reconstruction Using the Gluteus Maximus Muscle. Report of a Case. Dis Colon Rectum. $1985 ; 28: 973-7$.

4. Federov VD, Odaryuk TS, Shelygin YA, Tsarkov PV, Frolov SA. 1989. Method of creation of a smoothmuscle cuff at the site of the perineal colostomy after extirpation of the rectum. Dis Colon Rectum 32:562-566

5. Romano G, LaTorre F, Cutini G, Bianco F, Esposito P, Montori A. 2003. Total anorectal recon struction with the artificial bowel sphincter: report of eight cases. A quality-of-life assessment. Dis Colon Rectum $46: 730-734$

Figure legends

Figure 1 Anterior view of the neo-anus

Figure 2 Lateral view of the neo-anus

Figure 3 Gluteus maximus fasciculus in front of the rectum

Figure 4 Gluteus maximus fasciculus at back of the rectum 
Figure 5 Thickened prolapsed mucosa

Figure 6 Perineal view after surgery
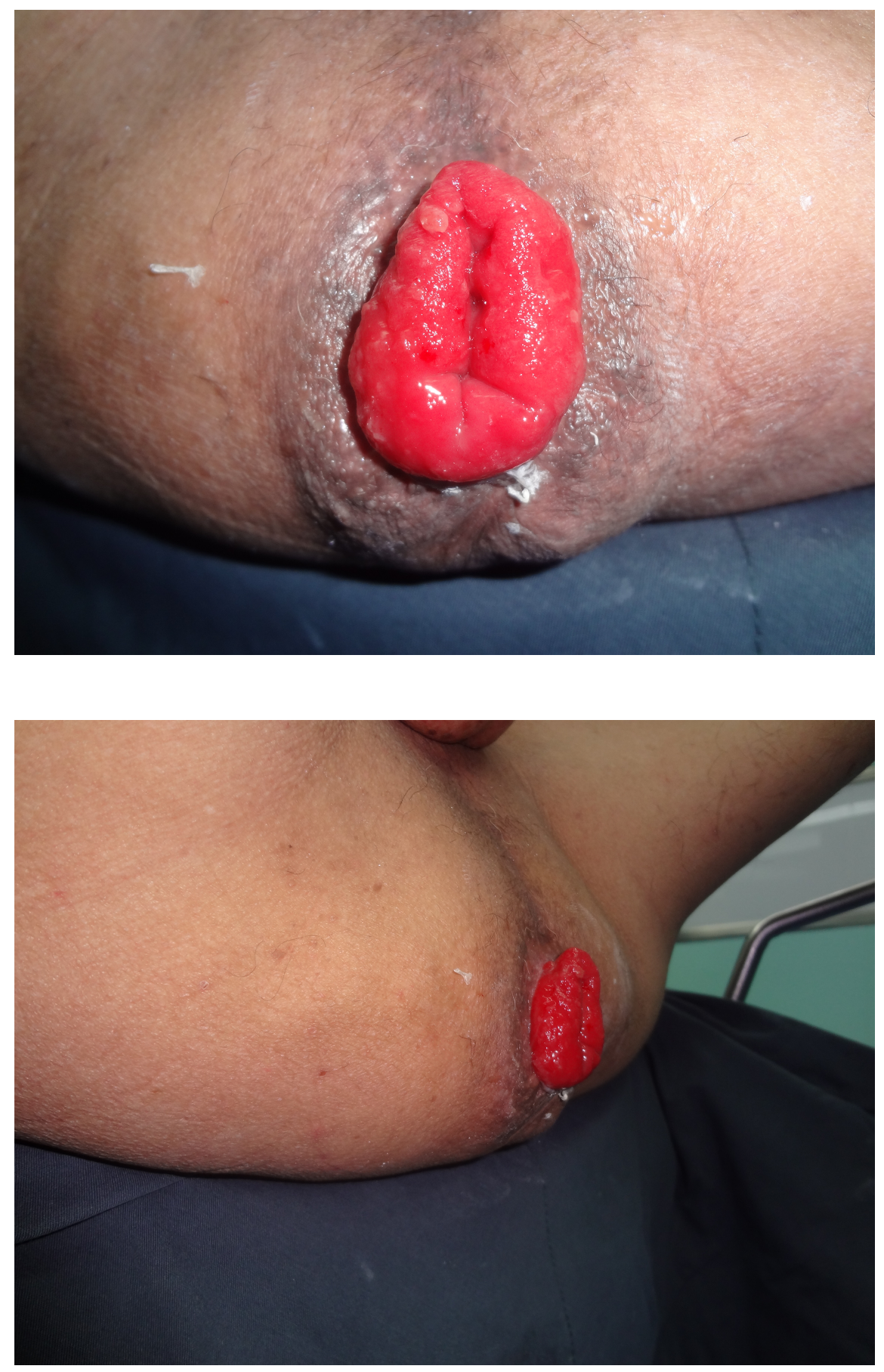

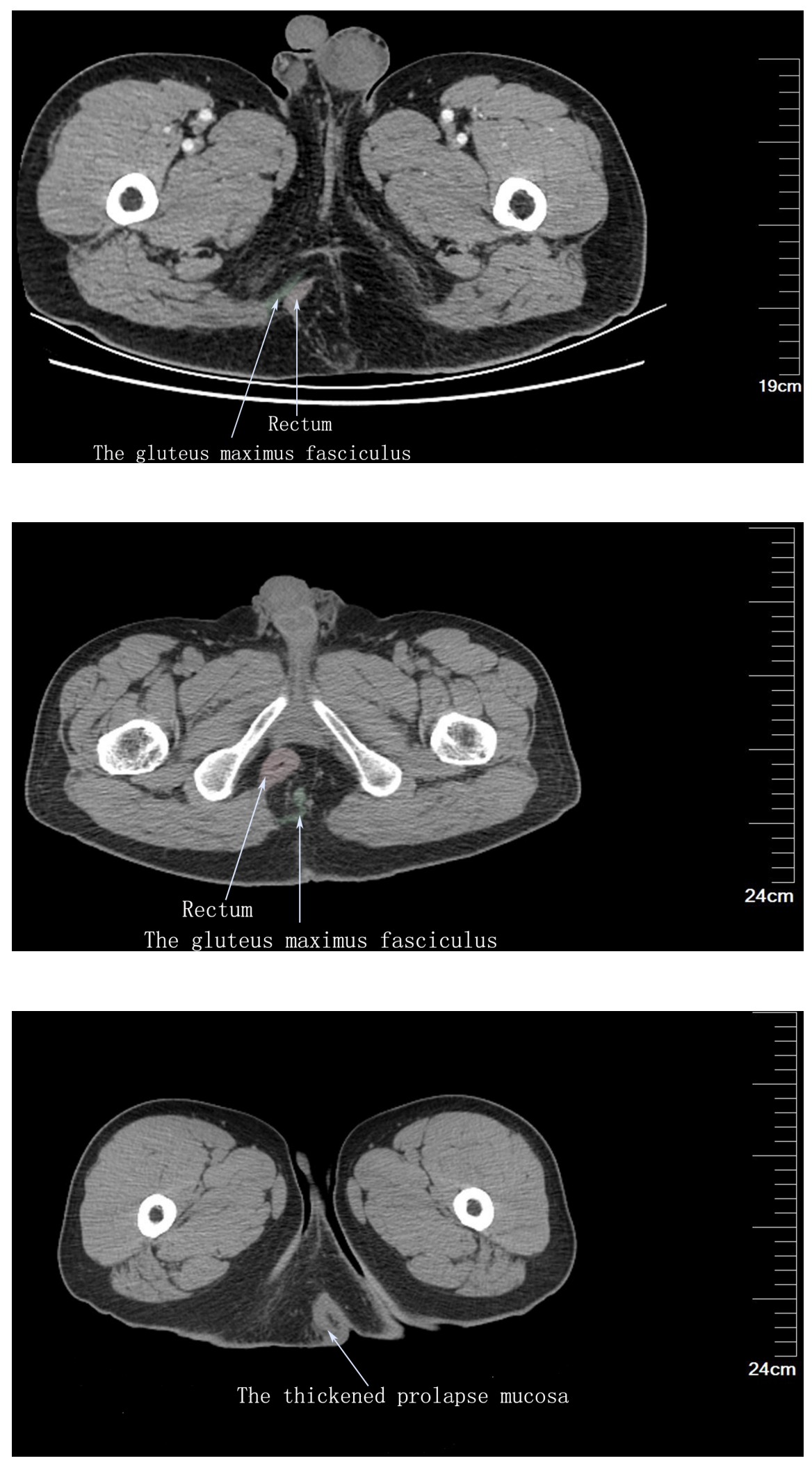


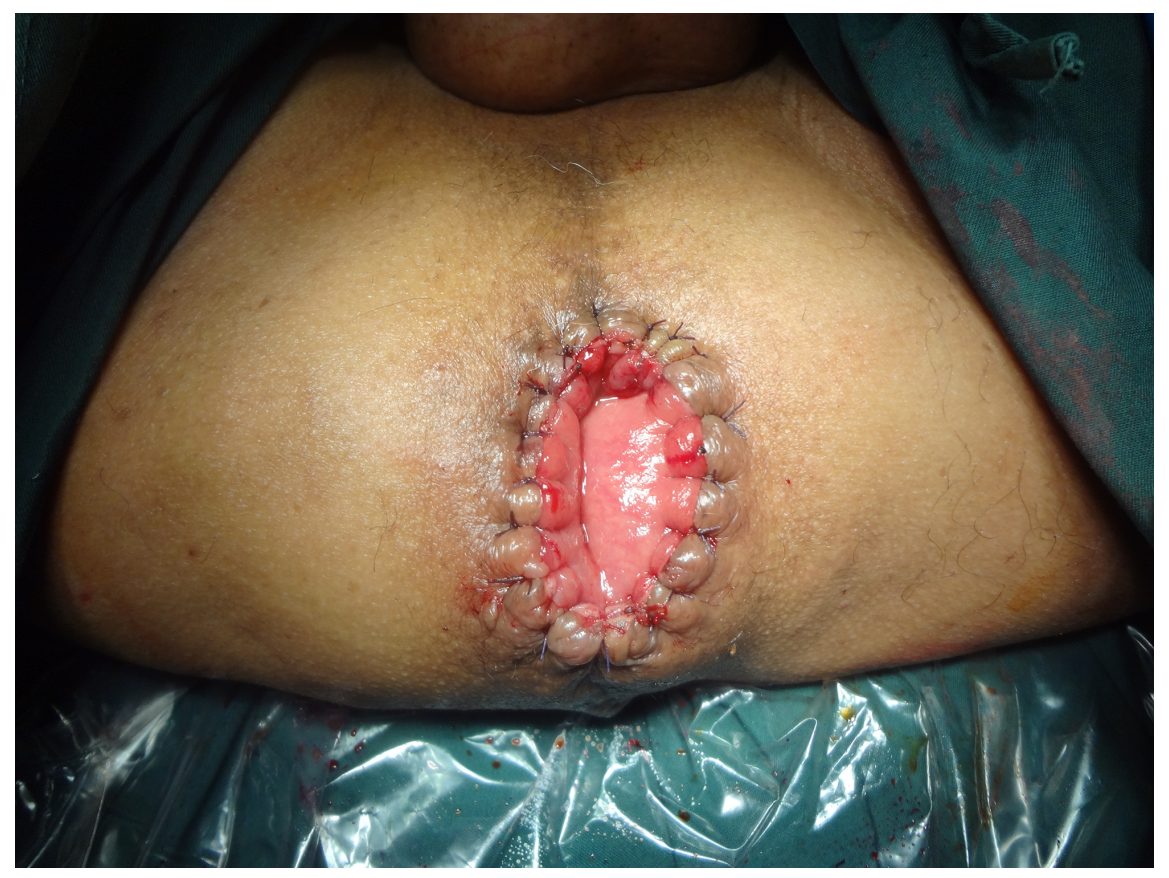

